\title{
Six-minute walk test (6MWT) in AL amyloidosis - baseline and 12 month follow-up after chemotherapy
}

Katerina Flatman, Darren Foard, Eleanor Pyart, Gaik Hughes, Randolph Gaudia, Charlene Kearney, Ismail Elmi, Alica Bangova, Pamela Libo-On, Joan Caringal-Galima, Emma Rowles, Christianne Guillotte, Philip Hawkins, Julian Gillmore and Thirusha Lane

National Amyloidosis Centre, Division of Medicine, Royal Free Campus, University College London, UK

Address for correspondence: Thirusha Lane, National Amyloidosis Centre, UCL Division of Medicine, Royal Free Campus, Rowland Hill Street, London, NW3 2PF. E-mail: t.lane@ucl.ac.uk

Background: The 6MWT provides a simple objective measure of functional exercise capacity [1], and is widely used as a marker of disease severity and response to therapy. We report here the change in functional exercise capacity, as measured by the $6 \mathrm{MWT}$, in patients with AL amyloidosis who have undergone chemotherapy.

Patients and methods: As part of our prospective observational study of chemotherapy in AL amyloidosis (ALchemy), we conducted 6MWT at diagnosis (baseline) and at 12 (range 9 -15) month follow-up, after patients had received chemotherapy. The 6MWT was conducted according to ATS guidelines [1]. Response to chemotherapy was assessed using the dFLC method [2]. Statistical analysis was performed using IBM SPSS 22.

Results: 402 patients performed a 6MWT at their baseline (diagnostic) visit. Of these, 203 had a $6 \mathrm{MWT}$ at 12 (range $9-15$ ) months. At baseline, the 6MWT distance, expressed as the percentage of age-corrected expected walking distance, correlated with many other established markers of disease (Table 1). Patients who achieved a complete response (CR) or very good partial response (VGPR) to chemotherapy by 12 months showed improved or stable 6MWT distance at 12 months, compared with those who had achieved only a partial response (PR) or no response (NR) to chemotherapy, whose walk distances were largely worse at 12 months (Figure 1). Baseline 6MWT distance was shown to be predictive of outcome in a Kaplan-Meier analysis: Median survival for those who walked $<50 \%$ of their expected distance was 5 months $(\mathrm{n}=86)$ and 38 months for those who walked $50-89 \%$ of their expected distance $(n=184)$. Median survival was not reached for those who walked $\geq$ $90 \%$ of their expected distance $(n=x)$.

Discussion and conclusions: The 6MWT at baseline provides a useful objective measure of patient functional capacity in AL amyloidosis and predicts survival. Achieving a CR or VGPR with chemotherapy typically results in improvement in functional capacity, in marked contrast to lesser degrees of clonal response. Further studies are planned.

\section{Correlation of baseline 6MWT with other prognostic factors}

\begin{tabular}{|l|l|l|l|}
\hline Variable & Test & Result & P-value \\
\hline Low BMI & Pearson & 0.127 & 0.016 \\
\hline Eligh FLC & Spearman's rank & -0.278 & $<0.001$ \\
\hline Systolic BP $<\mathbf{1 0 0}$ & Spearman's rank & 0.535 & $<0.001$ \\
\hline Elevated proteinuria & Pearson & 0.301 & $<0.001$ \\
\hline Elevated creatinine & Spearman's rank & 0.261 & $<0.001$ \\
\hline Cardiac involvement & Spearman's rank & -0.108 & 0.041 \\
\hline NYHA class & ANOVA & 53.395 & $<0.001$ \\
\hline
\end{tabular}


Table 1. Correlation of 6MWT distance with other prognostic factors. The baseline $6 \mathrm{MWT}$ result, expressed as the percentage of the expected walking distance for age, was compared to other clinical and biomarker prognostic factors. BMI and serum creatinine emerged as correlated $(\mathrm{p}<0.05)$ whilst high FLC concentration, high NT-proBNP, low systolic BP, the presence of cardiac amyloid and NYHA performance status were strongly correlated $(\mathrm{p}<0.005)$. ${ }^{*}$ Total body amyloid load as determined by SAP scintigraphy.

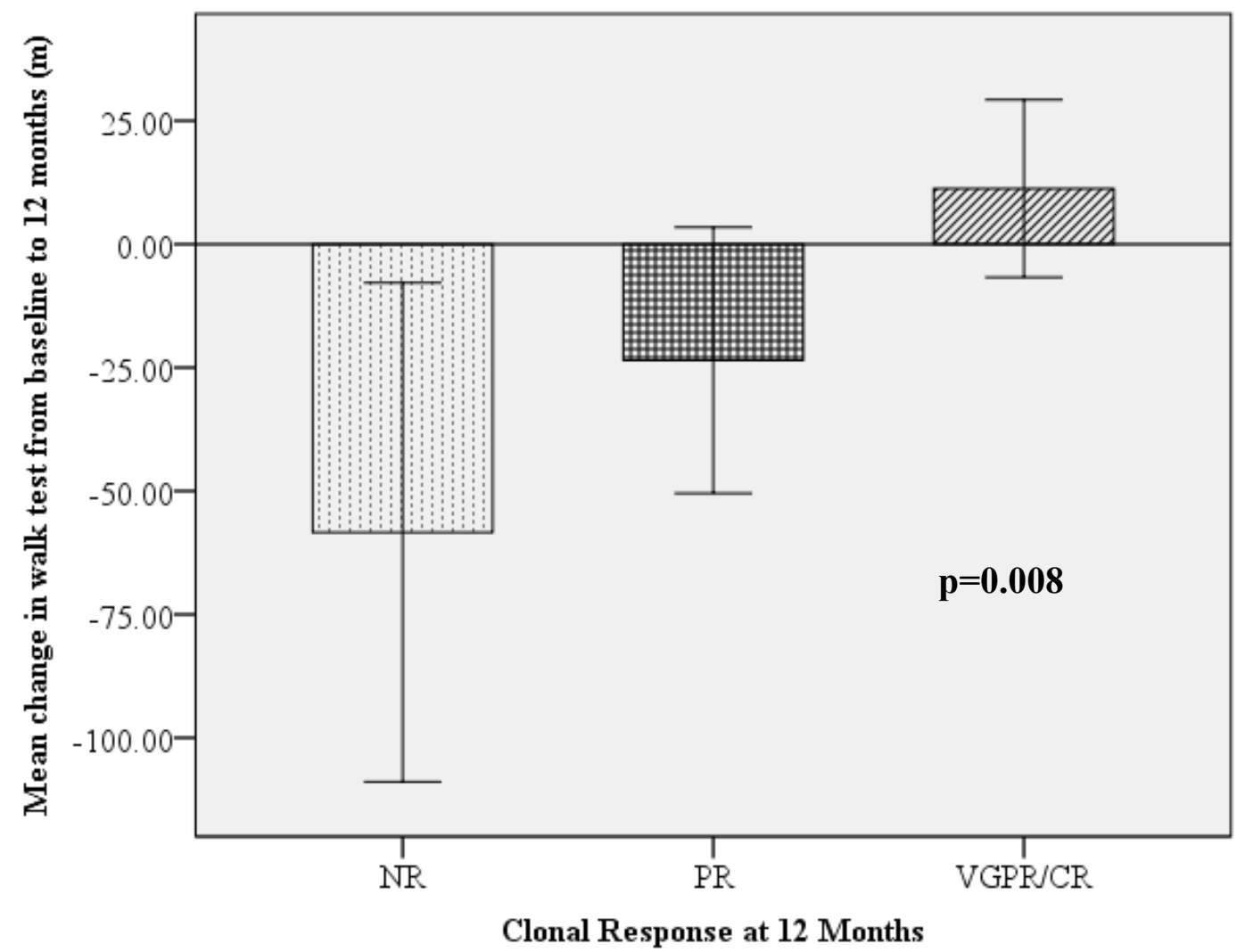

Error Bars: $95 \%$ CI

Figure 1. A good clonal response to chemotherapy is associated with stable or improved 6MWT distance at 12 months. This graph shows the mean change in 6MWT distance across clonal response groups. Patients who achieved a complete response (CR) or very good partial response (VGPR) by 12 months showed improved or stable 6MWT distance at 12 months, compared with those who had achieved only a partial response (PR) or no response (NR) to chemotherapy, whose walk distances were largely worse at 12 months (ANOVA $\mathrm{p}=$ 0.008; Error bars 95\% CI).

Declarations of interest: The authors have no conflicts to declare.

\section{References}

1. American Thoracic Society. ATS Statement: Guidelines for the Six-Minute Walk Test. Am J Respir Care Med 2002; 166:111-117.

2. Palladini G, Dispenzieri A, Gertz MA, Kumar S, Wechalekar A, Hawkins PN, et al. New criteria for response to treatment in immunoglobulin light chain amyloidosis based on free light chain measurement and cardiac biomarkers: impact on survival outcomes. J Clin Oncol. 2012;30(36):4541-9. 